
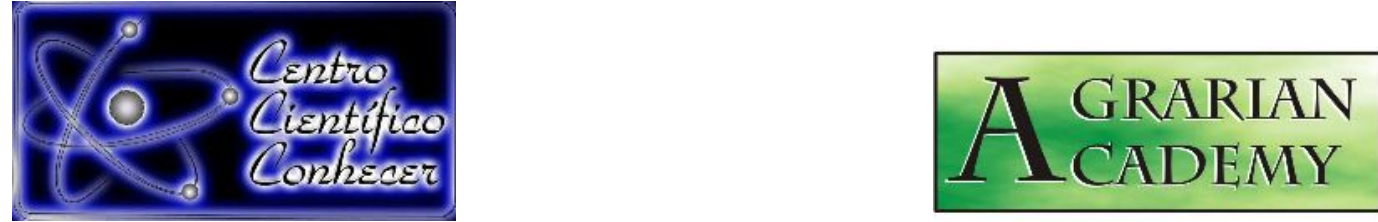

\title{
MICROCLIMA DE UM FRAGMENTO DE FLORESTA OMBRÓFILA ABERTURA NO SUL DA AMAZÔNIA
}

\author{
Charles Caioni \\ Professor da Universidade do Estado de Mato Grosso, Alta Floresta, Mato Grosso, \\ (charlescaioni@gmail.com)
}

Recebido em: 01/04/2020 - Aprovado em: 10/06/2020 - Publicado em: 30/07/2020

DOI: 10.18677/Agrarian_Academy_2020a10

\begin{abstract}
RESUMO
$\mathrm{Na}$ Amazônia Meridional grandes áreas de floresta nativa foram reduzidas a pequenos fragmentos florestais. Esses ambientes acabaram formando microclimas próprios, resultantes de processo biogeoquímicos que ocorrem dentro e fora do fragmento. Nessa conjetura, objetivou-se investigar a existência de diferença de algumas variáveis climáticas (temperatura e umidade do ar, velocidade do vento e temperatura do solo) dentro e fora de um fragmento de Floresta Ombrófila Aberta, localizado no Sudeste Amazônico. Para obtenção dos dados, inicialmente foi demarcado (via GPS Etrex H da Garmin) um transecto de 225 metros, considerando a borda do fragmento como ponto inicial e seu interior como ponto final. A partir do ponto inicial foram coletados (a cada 15 metros) dados de Temperatura e umidade do ar (Termo-Higro-Anemômetro Luxímetro Digital modelo: THAL-300). Também foi registrada a temperatura do solo a $10 \mathrm{~cm}$ de profundidade (via termômetro SP-B$4 \mathrm{H})$. A fim de comparar cada variável dentro e fora do fragmento, foram também realizadas coletas de temperatura do solo, temperatura do ar e umidade do ar, em um ponto fixo a 100 metros de distância da borda do fragmento. A temperatura do solo $\left(7,3^{\circ} \mathrm{C}\right)$ e do $\operatorname{ar}\left(6,4^{\circ} \mathrm{C}\right)$ foi menor dentro do fragmento. $O$ inverso foi observado para umidade relativa (10,6\%). Foi constatado ainda, um aumento linear da umidade do ar em função do distanciamento da borda. Esses resultados demonstram que o fragmento necessita da adoção de medidas de conservação, visto que o efeito de borda pode alterar a fauna e a flora local.
\end{abstract}

PALAVRAS-CHAVE: Amazônia Meridional, Desflorestamento, Umidade, temperatura.

\section{MICROCLIMATE OF A FRAGMENT OF OPENING SHOWER IN THE SOUTH AMAZON}

\section{ABSTRACT}

In the southern Amazon, large areas of native forest have been reduced to small forest fragments. These environments ended up forming their own microclimates, resulting from biogeochemical processes that occur inside and outside the fragment. In this conjecture, the objective was to investigate the existence of differences in some climatic variables (air temperature and humidity, wind speed and soil temperature) inside and outside a fragment of Open Rainforest, located in the Southeast Amazon. To obtain the data, a 225-meter transect was initially demarcated AGRARIAN ACADEMY, Centro Científico Conhecer - Jandaia-GO, v.7, n.13; p. 97 
(via GPS Etrex $\mathrm{H}$ from Garmin), considering the fragment's edge as a starting point and its interior as an end point. From the starting point, temperature and humidity data were collected (every 15 meters) (Thermo-Hygro-Anemometer Luxmeter Digital model: THAL-300). The soil temperature at $10 \mathrm{~cm}$ depth was also recorded (via thermometer SP-B-4H). In order to compare each variable inside and outside the fragment, collections of soil temperature, air temperature and air humidity were also carried out at a fixed point 100 meters away from the fragment's border. The soil $\left(7.3^{\circ} \mathrm{C}\right)$ and air $\left(6.4^{\circ} \mathrm{C}\right)$ temperature was lower inside the fragment. The reverse was observed for relative humidity $(10.6 \%)$. There was also a linear increase in air humidity due to the distance from the edge. These results demonstrate that the fragment requires the adoption of conservation measures, since the edge effect can alter the local fauna and flora.

KEYWORDS: Southern Amazon, Deforestation, Humidity, temperature.

\section{INTRODUÇÃO}

No sul da Amazônia, mais especificamente no estado de Mato Grosso, o desmatamento é um reflexo do modelo histórico desenvolvimentista e implantado na década de 70, mas, hoje, este pode ser encarado como um reflexo da economia nacional, que é pautada na produção de commodities, como soja, milho e carne bovina (MELLO; ARTAXO; 2017; COE et al., 2017; KHANNA et al., 2017; LISBOA et al., 2019). Nessa região ainda existem áreas de vegetação nativa, contudo, na maior parte fragmentadas e isoladas (CAIONI et al., 2018). O efeito de borda é um dos processos mais recorrentes nesses locais, ao ocasionar aumentos na incidência de luz e na temperatura da interface de transição entre a área florestal e a matriz não florestal, acaba provocando aumentos na evapotranspiração e consequentemente reduções na disponibilidade de água dentro do fragmento (NOVAIS et al., 2016; PSCHEIDT et al., 2018; COSTA et al., 2019). Essas novas condições podem funcionar como um filtro, selecionando espécies mais adaptadas as novas condições e assim alterando a fauna e flora local.

O microclima dentro de uma floresta é resultante tanto de processos bióticos quanto abióticos (BLUMENFELD et al., 2016; DIAS et al., 2019; GONÇALVES et al., 2019). Trocas energéticas que ocorrem ao redor do fragmento também podem influenciar, principalmente em variáveis como a temperatura e umidade. Bordas de fragmentos próximos a pastagens tendem a exibir temperaturas superiores quando comparados a florestas secundárias (SILVA et al., 2017). O tamanho e o formato do fragmento também exercem papel determinante, visto que o efeito de borda é menor em fragmentos maiores e de formato circular (MASSOLI et al., 2016).

Fragmentos próximos a rodovias tendem a serem mais antropizados (SILVA et al., 2017) devido a facilidade de acesso a esses locais. Em decorrência dessa condição, muitos desses ambientes exibem alto número de clareiras, o que facilita a entrada de luz e vento em seu interior, e por consequência, reduz a umidade do ar. Nessa conjectura, estudar o microclima desses locais é de grande relevância, pois possibilita entender qual o efeito da atividade humana sobre o microclima florestal.

Em meio a este contexto encontra-se um pequeno fragmento florestal localizado ao Sul da Amazônia, mais especificamente no município de Alta Floresta. Pertencente ao perímetro urbano do município, essa área vem sofrendo forte pressão antrópica. Nessa perspectiva, objetivou-se investigar a existência de diferença de algumas variáveis climáticas (temperatura e umidade do ar, velocidade do vento e temperatura do solo) dentro e fora de um fragmento de Floresta Ombrófila Aberta, localizado no Sudeste Amazônico. 


\section{Área de estudo}

\section{MATERIAL E MÉTODOS}

O estudo foi realizado em um fragmento de aproximadamente 20 hectares de Floresta Ombrófila Aberta (localizados em: 949'25"S a 950'30"S de latitude e $56^{\circ} 10^{\prime} 30$ "W a $56^{\circ} 09^{\prime} 10^{\prime \prime} W$ de longitude), pertencente ao município de Alta Floresta/MT, Sudeste Amazônico (Figura 1). A população do município é de 51.782 habitantes (IBGE, 2020).

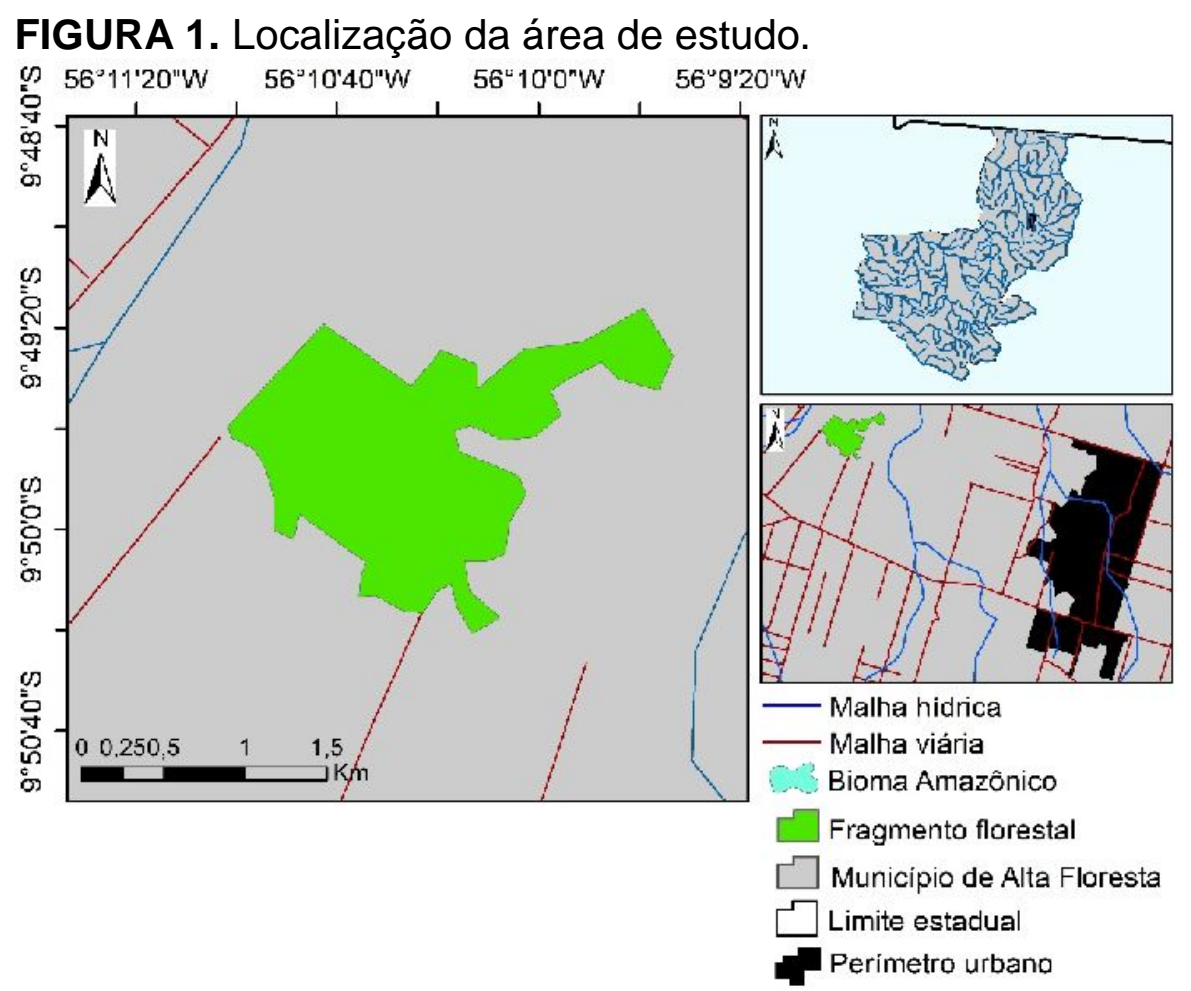

Fonte: próprio autor.

O clima da região é tropical de monções (Am), com duas nítidas estações: seca (de maio a setembro) e chuvosa (de outubro a abril do ano seguinte) (ALVARES et al., 2013). A média pluviométrica é de aproximadamente 2281 $\mathrm{mm} / \mathrm{ano}$, enquanto a temperatura fica em torno dos $25,4^{\circ} \mathrm{C}$ (CLIMATE-DATA.ORG, 2020). Agosto e setembro são os meses mais quentes do ano, com temperaturas próximas aos $26^{\circ} \mathrm{C}$ (OLIVEIRA et al., 2019).

\section{Procedimentos metodológicos}

A coleta de dados ocorreu em 3 de agosto de 2019, entre as 14:00 e 16:00 horas. Inicialmente foi demarcado (via GPS Etrex H da Garmin) um transecto de 225 metros, considerando a borda do fragmento como ponto inicial e seu interior como ponto final. A partir do ponto inicial foram coletados (a cada 15 metros) dados de velocidade do vento, temperatura e umidade do ar (via Termo-Higro-Anemômetro Luxímetro Digital modelo: THAL-300) e também temperatura do solo, a $10 \mathrm{~cm}$ de profundidade (via termômetro SP-B-4H). Em cada ponto de coleta foram realizadas três mensurações, com posterior obtenção de média. A fim de comparar cada variável dentro e fora do fragmento, foram também realizadas três coletas para cada variável, em um local fixo a 100 metros de distância da borda do fragmento.

Para identificar a existência de efeito de borda no fragmento foram realizadas regressões lineares entre a variável (temperatura e umidade ar, temperatura do solo e velocidade do vento) e sua respectiva distância com a borda do fragmento. 


\section{RESULTADOS E DISCUSSÕES}

Como um todo a temperatura do ar no fragmento foi de $30,2^{\circ} \mathrm{C}$ (Tabela 1). Esse resultado revela que embora o fragmento apresente clareiras em seu interior, 0 mesmo demonstrou $6,4^{\circ} \mathrm{C}$ a menos da temperatura registrada fora do fragmento. Esse resultado corrobora com a hipótese de que os ambientes florestais tendem a contribuir para a amenização da seca na Amazônia (CAIONI et al., 2020).

TABELA 1. Média de cada variável dentro e fora do fragmento

\begin{tabular}{|c|c|c|}
\hline Variável & Fragmento & $\begin{array}{l}\text { Fora do } \\
\text { fragmento }\end{array}$ \\
\hline Temperatura do ar & $30,2^{\circ} \mathrm{C}$ & $36,6^{\circ} \mathrm{C}$ \\
\hline Temperatura do solo & $30,6^{\circ} \mathrm{C}$ & $37,9^{\circ} \mathrm{C}$ \\
\hline Umidade do ar & $61,4 \%$ & $50,8 \%$ \\
\hline Velocidade do vento & $0,2 \mathrm{~m} / \mathrm{s}$ & $0,7 \mathrm{~m} / \mathrm{s}$ \\
\hline
\end{tabular}

Foi evidenciado um claro efeito de borda sobre a temperatura do ar, com maiores temperaturas na região próxima a borda do fragmento (Figura 2). Esse resultado está relacionado a borda receber maior incidência de luz e de ventos, visto que, muitos dos obstáculos naturais formados pelas árvores adjacentes já foram suprimidos (SILVA et al., 2017). O mesmo não ocorre no interior da floresta, onde densa vegetação possibilita que cerca de $80 \%$ da radiação incidente seja interceptada pelo dossel (NOVAIS et al., 2018). Esses resultados corroboram com Laurance et al. (2002) que mencionam que os reflexos do efeito de borda podem ser observados a mais de 400 metros da borda do fragmento. De modo geral, a existência de um gradiente de temperatura no fragmento é preocupante, visto que esse pode acarretar em mudanças na composição da fauna e da flora local.

FIGURA 2. Comportamento da temperatura da temperatura do ar e do solo ao longo do fragmento florestal (painel A). Relação entre temperatura do ar e do solo (painel B).
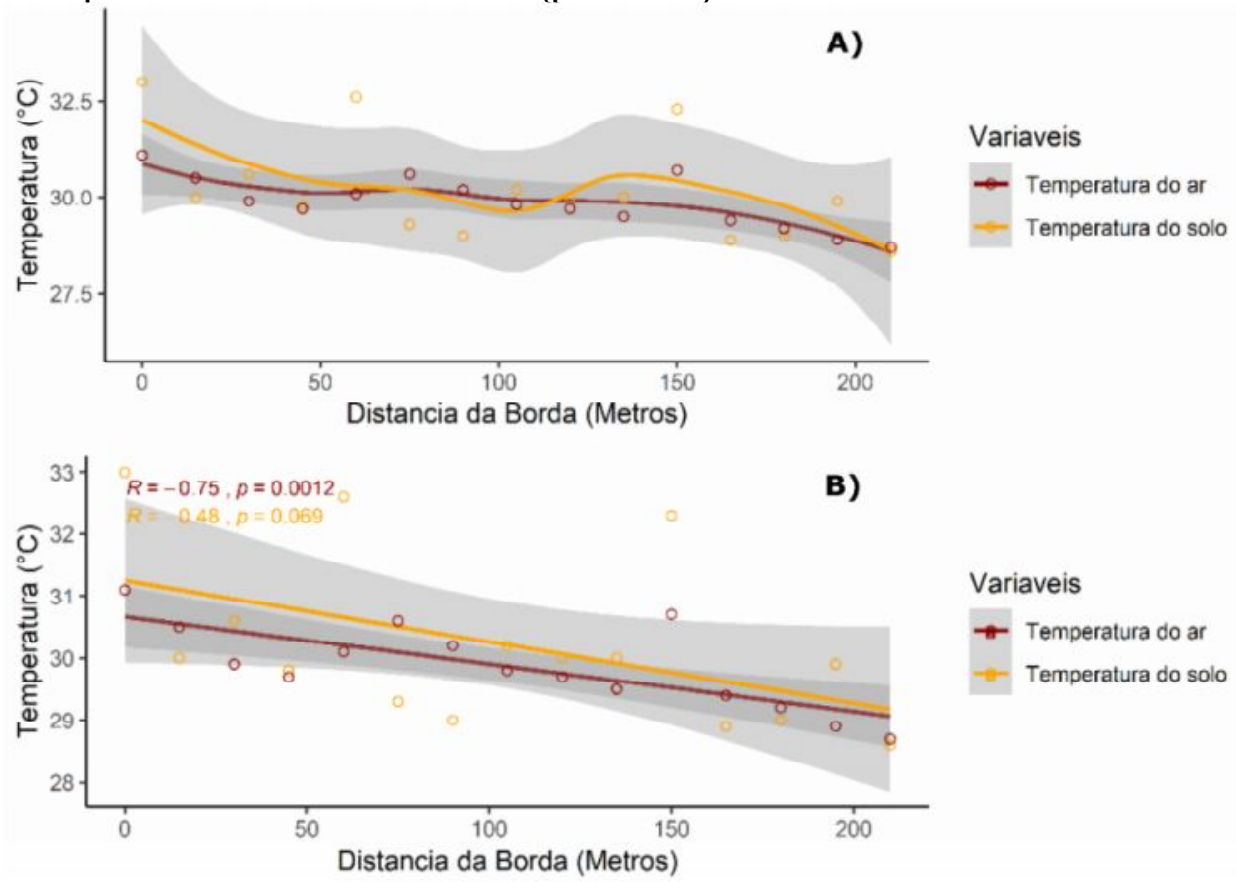

Fonte: próprio autor. 
Embora a temperatura do solo tenha exibido um decréscimo para estimativas mais próximas ao centro do fragmento, essa redução não foi significava aos $10 \mathrm{~cm}$ de profundidade. Esse resultado pode estar relacionado ao fragmento possuir aberturas no dossel que permitem que a luz alcance diretamente o solo da floresta. Embora esse processo esteja ocorrendo, observou-se uma diferença de $7,3^{\circ} \mathrm{C}$ entre a temperatura de dentro e de fora do fragmento. Essa diferença pode ser atribuída a serapilheira, que forma uma camada que protege o solo contra a exposição direta a radiação incidente, e assim, mantém a umidade e a estabilidade térmica do solo.

A umidade relativa do ar registrada no fragmento foi superior $(10,6 \%)$ a registrada fora do fragmento. A umidade também demonstrou um padrão ascendente à medida que se adentrou no fragmento florestal (Figura 3), possivelmente devido a maior incidência de luz e ventos na borda. Essa hipótese é apoiada pelos registros de velocidade do vento que foi de $0,5 \mathrm{~m} / \mathrm{s}$ para o primeiro ponto de coleta e de $0,1 \mathrm{~m} / \mathrm{s}$ para o último ponto, a 225 metros da borda. Esse padrão também foi constatado por Muller et al., (2009), que observaram uma tendência de aumento da umidade para áreas mais próximas ao centro do fragmento. Outro processo que pode estar ocorrendo é o fechamento estomático das plantas, visto que a fim de evitar a perda excessiva de água para a atmosfera as plantas fecham os estômatos reduzindo a taxa de evapotranspiração (FURQUIM et al., 2018).

FIGURA 3. Umidade relativa do ar aferida ao longo do transecto
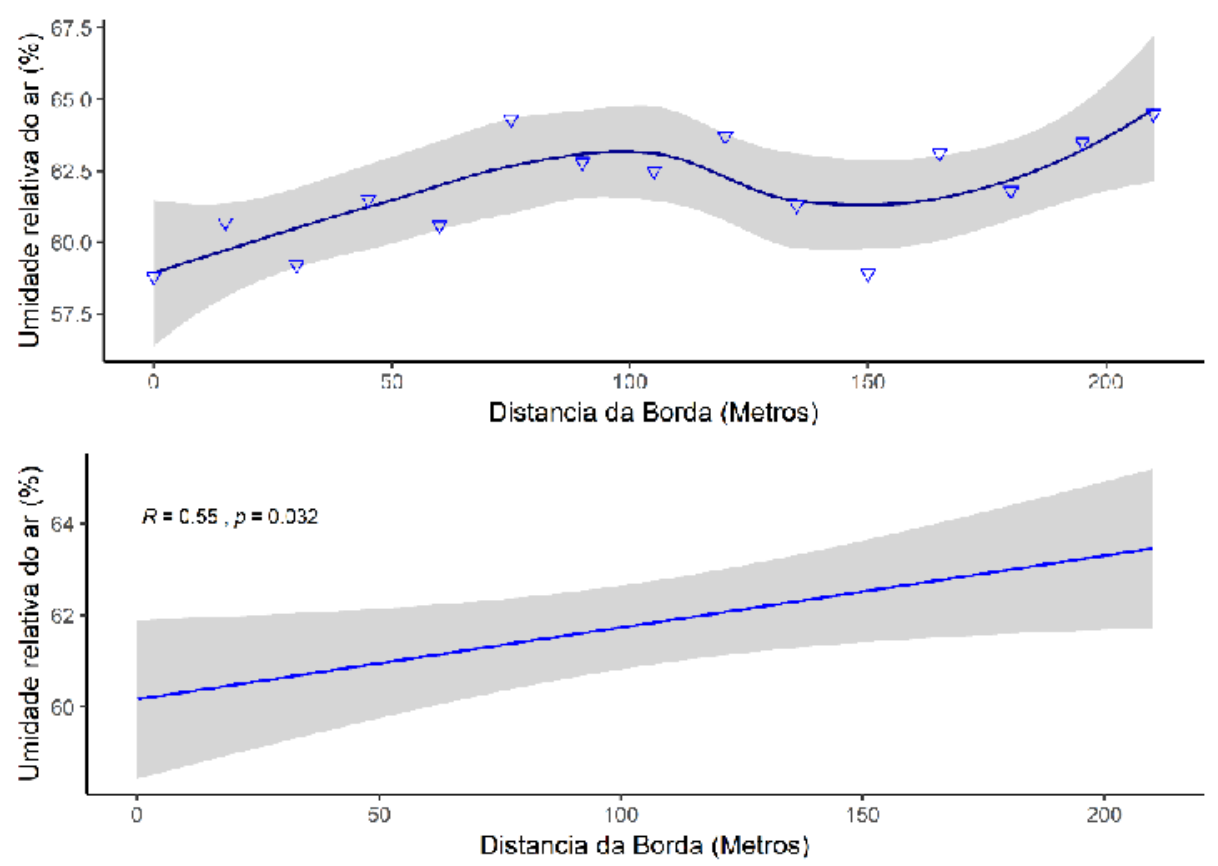

Fonte: próprio autor.

Próximo aos 150 metros de distância da borda, a umidade relativa também demonstrou uma ligeira redução, seguido de um aumento da temperatura do ar, isso se deve ao surgimento de uma pequena clareira no transecto, característica de fragmentos em processo de degradação. Essa explicação é apoiada pela relação inversa observada entre a temperatura e umidade do ar (Figura 4). Esse resultado também corrobora com Bazzaz e Pickett (1980) que afirmaram que nas áreas de 
clareira as condições ambientais diferem daquelas dos dosséis fechados, visto que variáveis como, luz, temperatura e umidade, deixam de ser controlados pelos indivíduos dos dosséis dominantes.

FIGURA 4. Relação inversa entre a umidade relativa e a temperatura do ar no fragmento florestal.

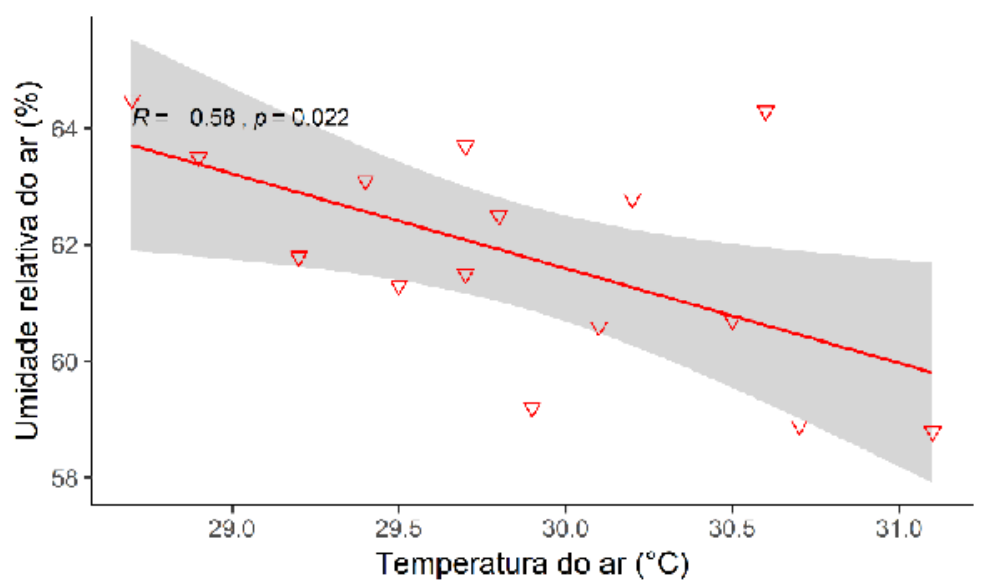

Fonte: próprio autor.

\section{CONCLUSÕES}

Evidenciamos diferença dentro e fora do fragmento para todas as variáveis climáticas estudadas. A temperatura (do ar e do solo) e a velocidade do vento foram inferiores dentro do fragmento florestal. $O$ inverso ocorreu com a umidade relativa do ar. Constatou-se ainda, uma relação inversa entre a temperatura e a umidade relativa do ar registrada dentro do fragmento.

Mediante as áreas mais próximas da borda do fragmento terem apresentado menor umidade relativa do ar e maiores temperaturas (do solo e ar), ficou evidente que o efeito de borda ao longo dos anos contribuiu para mudanças no microclima florestal e consequentemente na biota local. É esperado que este processo venha a aumentar sua intensidade no futuro, deixando o ambiente mais seco, e assim extinguindo as espécies menos adaptadas as novas condições microclimáticas. Desta forma, sugere-se a adoção de medidas de conservação, a fim de manter a fauna e flora local.

\section{REFERÊNCIAS}

ALVARES, C.A., STAPE, J.L., SENTELHAS, P.C., GONÇALVES, J.L.M.; SPAROVEK, G. Köppen's climate classification map for Brazil. Meteorologische Zeitschrift, v.22, n.6, p.711-728, 2013. Disponível em: < https://www.schweizerbart.de/papers/metz/detail/22/82078/Koppen_s_climate_classif ication_map_for_Brazil?af=crossref. doi.org/10.1127/0941-2948/2013/0507 > Acesso em: 03 nov. $201 \overline{9}$.

BAZZAZ, E. A.; PICKETT, S. T. A. Physiological ecology of tropical succession: A comparative review. Annual Review of Ecology, v.11, p. 287-310, 1980. Disponível em: <https://www.jstor.org/stable/2096910?seq=1 > Acesso em: 30 out. 2019. 
BLUMENFELD, E. C.; DOS SANTOS, R. F.; THOMAZIELLO, S. A.; RAGAZZI, S. Relações entre tipo de vizinhança e efeitos de borda em fragmento florestal. Ciência Florestal, v. 26, n. 4, p. 1301-1316, 2016. Disponível em: < https://periodicos.ufsm.br/cienciaflorestal/article/view/25150.

http://dx.doi.org/10.5902/1980509825150 > Acesso em: 08 mar. 2020.

CAIONI, C.; NEVES, S. M. A. S.; COCHEV, J. S. Análise da temperatura aparente dos elementos da paisagem da microbacia Pedra do Índio em Alta FlorestaMT. Semina: Ciências Exatas e Tecnológicas, v. 39, n. 1, p. 11-18, 2018. Disponível em: http://www.uel.br/revistas/uel/index.php/semexatas/article/view/26057.

http://dx.doi.org/10.5433/1679-0375.2018v39n1p11 > Acesso em: 04 mai. 2020.

CAIONI, C.; SILVÉRIO, D. V.; MACEDO, M. N.; COE, M. T.; BRANDO, P. M. Droughts Amplify Differences Between the Energy Balance Components of Amazon Forests and Croplands. Remote Sensing, v. 12, n. 3, p. 525, 2020. Disponível em: < https://www.mdpi.com/2072-4292/12/3/525. 10.3390/rs12030525 > Acesso em: 21 mai. 2020.

CLIMATE-DATA.ORG. Clima: Alta Floresta. Disponível em: < https://pt.climatedata.org/america-do-sul/brasil/mato-grosso/alta-floresta-4078/ >. Acesso em: 01 jun. 2020.

COE, M. T.; BRANDO, P. M.; DEEGAN, L. A.; MACEDO, M. N.; NEILL, C. SILVÉRIO, D. V. The forests of the Amazon and Cerrado moderate regional climate and are the key to the future. Tropical Conservation Science, v. 10, p. 1940082917720671, 2017. Disponível em: < https://journals.sagepub.com/doi/full/10.1177/1940082917720671. https://doi.org/10.1177/1940082917720671 > Acesso em: 03 nov. 2019.

COSTA, A.; GALVÃO, A.; SILVA, L. G. Mata Atlântica brasileira: análise do efeito de borda em fragmentos florestais remanescentes de um hotspot para conservação da biodiversidade. Revista de Geografia, Meio Ambiente e Ensino, v. 10, n. 1, p. 112123, 2019. Disponível em: < http://www.fecilcam.br/revista/index.php/geomae/article/view/415> Acesso em: 08 mar. 2020.

DIAS, P. B.; MOREIRA, L. N.; SILVA, G. F.; PEZZOPANE, J. E. M.; DIAS, H. M. Riqueza, estrutura e relações ambientais em uma Floresta Nacional no Sudeste do Brasil. Revista Brasileira de Ciências Agrárias (Agrária), v. 14, n. 4, p. 6897, 2019. Disponível em: < http://www.agraria.pro.br/ojs2.4.6/index.php?journal=agraria\&page $=$ article $\& o p=v i e w \& p a t h \% 5 B \% 5 D=a g r a r i a \_v 14 i$ 4a6897. 10.5039/agraria.v14i4a6897 > Acesso em: 30 out. 2019.

FURQUIM, L. C.; DOS SANTOS, M. P.; DE ANDRADE, C. A. O.; DE OLIVEIRA, L. A.; EVANGELISTA, A. W. P. Relação entre plantas nativas do Cerrado e água. Científic@-Multidisciplinary Journal, v. 5, n. 2, p. 146-156, 2018. Disponível em: < http://periodicos.unievangelica.edu.br/index.php/cientifica/article/view/2553. https://doi.org/10.29247/2358-260X.2018v5i2.p146-156 > Acesso em: 03 nov. 2019. 
GONÇALVES, A. L.; CRUZ, V. M. S.; SERRA, A. B. Fragmentação florestal na Região de Integração do Lago de Tucuruí, Pará, Brasil. Revista de Ciências Agrárias Amazonian Journal of Agricultural and Environmental Sciences, v. 62, 2019. Disponível em: < http://ajaes.ufra.edu.br/index.php/ajaes/article/view/3048. http://dx.doi.org/10.22491/rca.2019.3048> Acesso em: 08 mar. 2020.

IBGE. Instituto Brasileiro de Geografia e Estatística. Cidades. Disponível em: $<$ https://cidades.ibge.gov.br/brasil/mt/alta-floresta/panorama>. Acesso em: 01 Jun. 2020.

KHANNA, J.; MEDVIGY, D.; FUEGLISTALER, S.; WALKO, R. Regional dry-season climate changes due to three decades of Amazonian deforestation. Nature Climate Change, v. 7, n. 3, p. 200-204, 2017. Disponível em: < https://www.nature.com/articles/nclimate3226. https://doi.org/10.1038/nclimate3226> Acesso em: 30 out. 2019.

LAURANCE, W. F.; LOVEJOY, T. E.; VASCONCELOS, H. L.; BRUNA, E. M.; DIDHAM, R. K.; STOUFFER, P. C.; SAMPAIO, E. Ecosystem decay of Amazonian forest fragments: a 22 year investigation. Conservation Biology, v. 16, n. 3, p. 605618, 2002. Disponível em: Acesso em: 17 mar. 2020.

LISBOA, L. S.; ALMEIDA, A. S.; LAMEIRA, W. J. Análise temporal da fragmentação florestal no leste da Amazônia legal. Novos Cadernos NAEA, v. 22, n. 3, 2019. Disponível em: < https://conbio.onlinelibrary.wiley.com/doi/10.1046/j.15231739.2002.01025.x. 10.1046/j.1523-1739.2002.01025.x> Acesso em: 21 jan. 2020.

MASSOLI, J. V.; STATELLA, T.; SANTOS, V. S. Estimativa da fragmentação florestal na microbacia Sepotubinha, Nova Marilândia - MT, entre os anos de 1990 a 2014. Caminhos de Geografia, v. 17, n. 60, p. 480-60, 2016. Disponível em: < http://www.seer.ufu.br/index.php/caminhosdegeografia/article/view/31859.

https://doi.org/10.14393/RCG176004> Acesso em: 30 out. 2019. Disponível em: < > Acesso em: 21 jan. 2020

MELLO, N. G. R.; ARTAXO, P. Evolução do plano de ação para prevenção e controle do desmatamento na Amazônia legal. Revista do Instituto de Estudos Brasileiros, n. 66, p. 108-129, 2017. Disponível em: < https://www.scielo.br/scielo.php?pid=S0020-

$38742017000100108 \&$ script $=$ sci_abstract\&tIng $=$ pt.

https://doi.org/10.11606/issn.2316-901x.v0i66p108-129> Acesso em: 17 mar. 2020.

MULLER, A.; BATAGHIN, F. A.; SANTOS, S. C. Efeito de borda sobre a comunidade arbórea em um fragmento de Floresta Ombrófila Mista, Rio Grande do Sul, Brasil. Perspectiva, Erechim, v. 34, 2009. Disponível em: < http://www.uricer.edu.br/site/pdfs/perspectiva/125_72.pdf> Acesso em: 21 jan. 2020.

NOVAIS, D. B.; FERREIRA, J. S.; BARRETO, P. A. B. Fertilidade do solo como indicador do efeito de borda em fragmento florestal, Vitória da Conquista, Bahia. Revista Verde de Agroecologia e Desenvolvimento Sustentável, v. 11, n. 4, p. 185-189, 2016. Disponível em: < 
https://www.gvaa.com.br/revista/index.php/RVADS/article/view/4081.

https://doi.org/10.18378/rvads.v11i4.4081 > Acesso em: 17 mar. 2020.

NOVAIS, J. W. Z.; SANCHES, L.; DE MORAES DIAS, V. R.; MACHADO, N. G.; DA SILVA, L. B.; AQUINO, A. M. Variação espaço-temporal da PAR refletida pelo solo e transmitida pelo dossel em floresta inundável no Pantanal mato-grossense. Ciência Florestal, v. 28, n. 4, p. 1502-1513, 2018. Disponível em: < https://periodicos.ufsm.br/cienciaflorestal/article/view/35097.

http://dx.doi.org/10.5902/1980509835097>. Acesso em: 21 jan. 2020.

PSCHEIDT, F.; HIGUCHI, P.; SILVA, A. C. D.; RECH, T. D.; SALAMI, B.; FERREIRA, T. D. S.; BENTO, M. A. Efeito de borda como fonte da heterogeneidade do componente arbóreo em uma floresta com araucárias no Sul do Brasil. Ciência Florestal, v. 28, n. 2, p. 601-612, 2018. Disponível em: < https://www.scielo.br/scielo.php?script=sci_arttext\&pid=S1980-50982018000200601 doi.org/10.5902/1980509832046>. Acesso em: 17 mar. 2020.

OLIVEIRA, A. S.; PIERANGELI, M. A. P.; SOUSA, J. B. Características do meio físico da bacia hidrográfica do médio curso do rio Teles Pires, no município de Alta Floresta-MT. Revita Equador, v. 8, n. 3, p. 159-177, 2019. Disponível em: < https://periodicos.ufsm.br/cienciaflorestal/article/view/32046. 10.5902/19805098 > Acesso em: 04 mai. 2020.

SILVA, V. L.; MALLMANN, I. T.; CUNHA, S.; SCHMITT, J. L. Impacto do efeito de borda sobre a comunidade de samambaias epifíticas em Floresta com Araucária. Revista Brasileira de Ciências Ambientais (Online), n. 45, p. 19-32, 2017. Disponível em: http://rbciamb.com.br/index.php/Publicacoes_RBCIAMB/article/view/93. https://doi.org/10.5327/Z2176-947820170229> Acesso em: 17 mar. 2020. 